\title{
Image Compression: A Comparative Study between ANN and Traditional Approach
}

\author{
Aman Utkarsh \\ Sikkim Manipal Institute of Technology \\ Sikkim, India
}

\author{
Chandralika Chakraborty \\ Sikkim Manipal Institute of Technology \\ Sikkim, India
}

\begin{abstract}
Image compression is a highly essential part of image processing and is a necessity of the modern world required in various fields. It is a process of representing image data using fewer bits than it is required for the original, by performing image compression a certain amount of data used by the image for its storage can be reduced. Compression is necessary in cases where a large amount of data is to be stored or transferred.
\end{abstract}

This paper reviews some of the conventional methods for achieving Image compression, viz. Run length encoding, DCT, DWT to name a few. Artificial neural networks can also be used to achieve image compression. Here, an attempt is made to compare between the traditional methods of performing image compression and the artificial neural network approach.

\section{Keywords}

Image Compression, Run-length encoding, DCT, DWT, Levenberg-Marquardt.

\section{INTRODUCTION}

Image compression is an essential procedure in the modern world where there is a requirement of storing large amount of data using as much less storage space as possible. Image compression can be achieved using various algorithms which may be divided into lossless and lossy categories. In lossless compression scheme, the reconstructed image after compression, is numerically identical to the original image whereas in lossy compression techniques the compression ratio is high. The decompressed image is not exactly identical to the original image, but close to it. Removal of redundant data is a very important part of image compression. There are three types of redundancies, which are, coding redundancy, which is present when less than optimal code words are used, interpixel redundancy, which results from correlations between the pixels of an image \& psycho visual redundancy which is due to data that is ignored by the human visual system (i.e. visually nonessential information). These are the conventional techniques used for image compression.

Apart from the conventional techniques artificial neural network can also be used to achieve image compression. ANN is an efficient method to perform image compression and is widely used in the modern world.

\section{COMPRESSION TECHNIQUES}

There are basically two types of compression techniques, lossless and lossy. When lossless data is decompressed, the resulting image is identical to the original whereas lossy compression algorithms result in loss of data and the decompressed image may not be exactly the same as the original. Some of the lossless compression techniques are:
- $\quad$ Entropy encoding

- Huffman encoding

- Arithmetic coding

- $\quad$ LZW coding

Some of the lossy compression techniques are:

- Transform coding

- $\quad$ DCT

- DWT

- Fractal Compression

Artificial neural network approaches are widely used now-adays for the procedure of image compression. Artificial neural networks are simplified models of the biological neuron system. A neural network is a highly interconnected network with a large number of processing elements called neurons in an architecture inspired by the brain. The various architectures of an artificial neural networks can be classified into the following categories :

- Back Propagation Neural Network

- Multi-layer Feed Forward Artificial Neural Network

- Multilayer Perception

- Self-organizing maps

In this work the following four techniques are implemented :

- $\quad$ Run-length encoding (RLE)

- $\quad$ Block truncation coding (BTC)

- Discrete cosine transformation (DCT)

- Levenberg-Marquardt algorithm (LM)

The comparision among the above four techniques are as shown in Table 2. Run length encoding is a lossless technique, block truncation coding and discrete cosine transformation are lossy techniques and Levenberg-Marquardt algorithm is an ANN algorithm.

\section{IMAGE COMPRESSION \\ 3.1 Run length encoding}

RLE is one of the simple data compression algorithms. In RLE data occurs in a sequences of runs. In runs same data values occur together and can be repeated at many instances. RLE is the process to compress those runs into a minimal value so as to represent the continuous values in minimum space possible.

- $\quad$ Run length encoding 


\subsubsection{Input and Output}

The following is the output obtained using the run length encoding algorithm for image compression

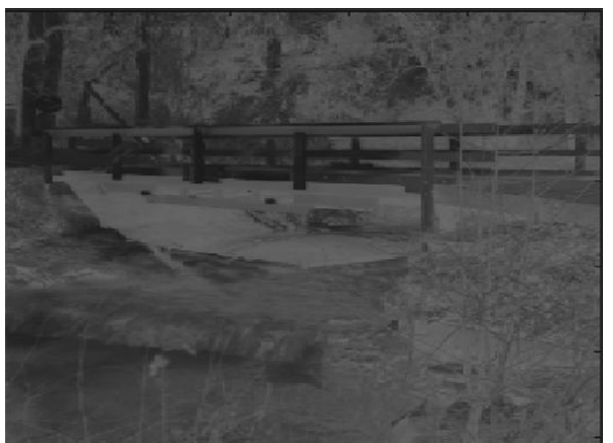

Figure 1: Original (Input) image

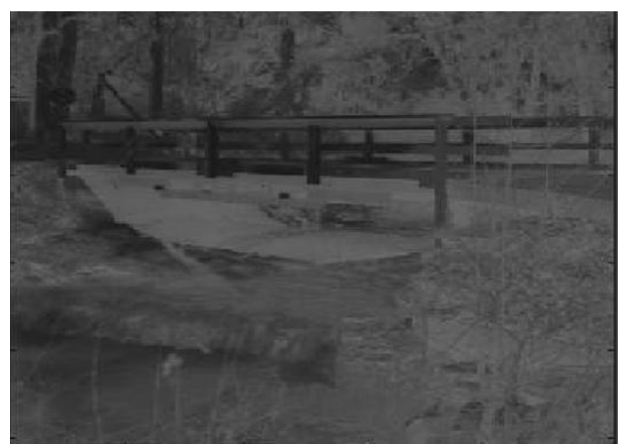

Figure 2: Compressed (Output) image

\subsection{Block Truncation Coding}

\section{$[1,2]$}

Block truncation coding is a lossy compression technique. Lossy compression techniques deliberately introduce a certain amount of distortion to the encoded image. These techniques must find an appropriate balance between the amount of error (loss) and the resulting bit savings.

A $\mathbf{J} \times \mathbf{J}$ pixel image is divided into blocks of typically $\mathrm{m} \times \mathrm{m}$ pixels, where $\mathrm{m}<\mathrm{J}$. For each block the mean and standard deviation are calculated, these values change from block to block. These two values define what values the reconstructed or new block will have, in other words the blocks of the BTC compressed image will all have the same mean and standard deviation of the original image [2].

\subsubsection{Input and Output}

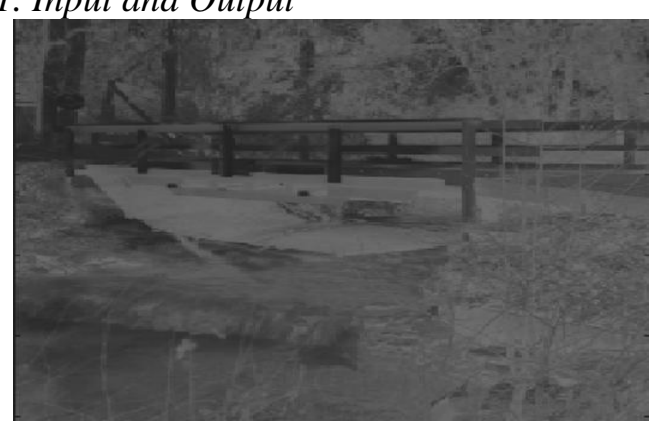

Figure 3: Original (Input) image for block truncation coding.

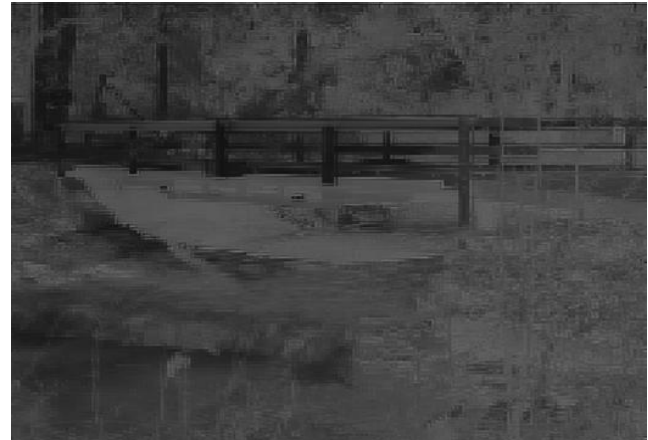

Figure 4: Compressed (Output) image obtained for block truncation coding

\subsection{Discrete Cosine}

\section{Transformation $[1,3]$}

The Discrete cosine transformation process is applied on blocks of $\mathrm{m} \times \mathrm{m}$ pixels, which will convert into series of coefficients, which define spectral composition of the block. The transformer transforms the input data into a format to reduce interpixel redundancies in the input image. Transform coding techniques use a reversible, linear mathematical transform to map the pixel values onto a set of coefficients, which are then quantized and encoded.

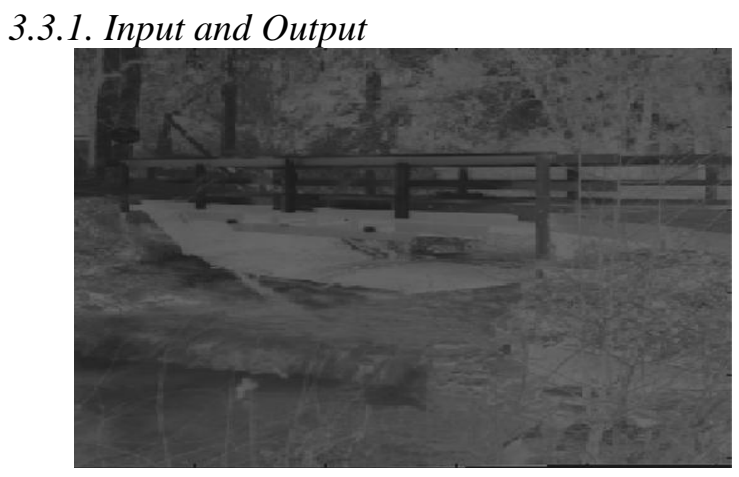

Figure 5: Original (Input) image for discrete cosine transformation

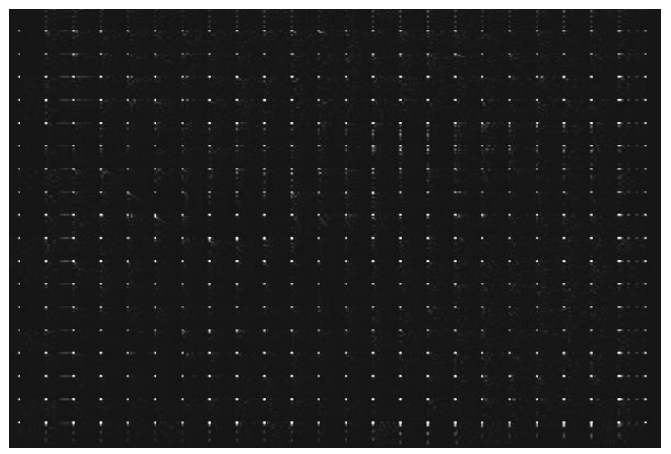

Figure 6: Compressed (Output) image (8X8 DCT) obtained for discrete cosine transformation 


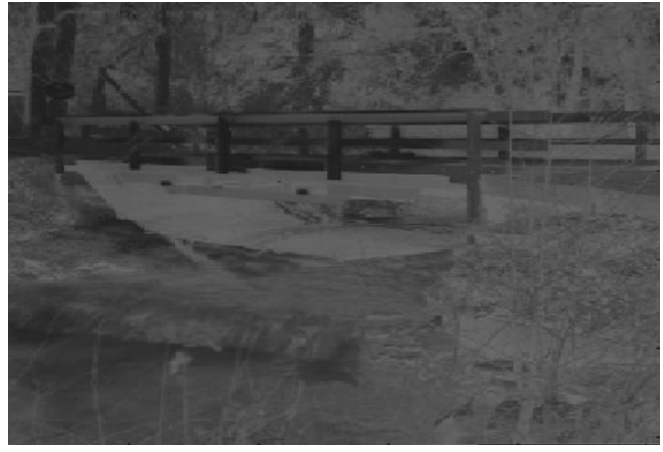

Figure 7: Decompressed (reconstructed) image obtained for discrete cosine transformation

\subsection{Levenberg-Marquardt Algorithm}

\section{$[4,5,6]$}

Levenberg-Marquardt algorithm uses a different back propagation artificial neural networks, which are used as compressor and de-compressor and it is achieved by dividing the image in to blocks, computing the complexity of each block and then selecting one network for each block according to its complexity value.

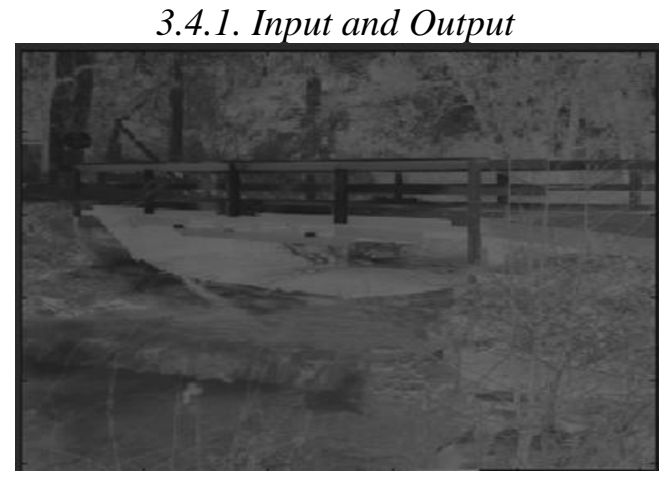

Figure 8: Original (input) image for levenberg-marquardt algorithm

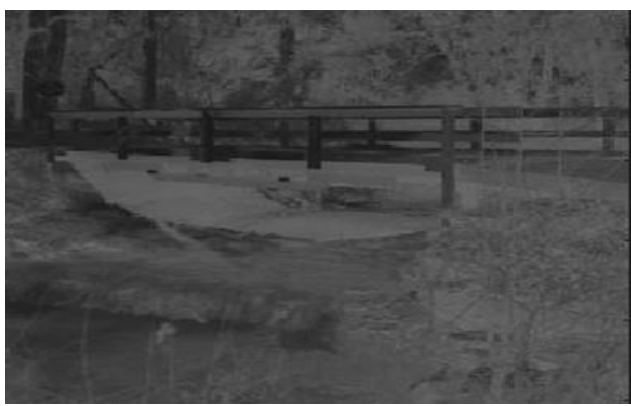

Figure 9: Compressed (Output) image obtained for Levenberg-Marquardt algorithm

\section{COMPARISON BETWEEN VARIOUS ALGORITHMS USED FOR COMPRESSION}

The Levenberg-Marquardt algorithm is implemented in MATLAB and the algorithm's peak signal to noise ratio, root mean square error, and is obtained with respect to various parameters - PSNR, RMSE and BP, for different number of iterations.
Table 1: Value of different parameters at different iterations

\begin{tabular}{|c|c|c|c|}
\hline \multicolumn{4}{|c|}{ LEVENBERG-MARQUARDT ALGORITHM } \\
\hline $\begin{array}{c}\text { NO. OF } \\
\text { ITERATIONS }\end{array}$ & PSNR & RMSE & BP \\
\hline $\mathbf{1 1 0 0}$ & 22.4320 & 24.1487 & 0.8472 \\
\hline $\mathbf{1 3 0 0}$ & 22.5294 & 24.4339 & 0.8491 \\
\hline $\mathbf{1 6 2 0}$ & 22.8409 & 21.7741 & 0.8498 \\
\hline $\mathbf{2 2 0 0}$ & 22.8587 & 23.2767 & 0.8554 \\
\hline
\end{tabular}

The efficiency of different image compression algorithms used in this work can be computed using various parameters. The values obtained using the various parameters helps to determine the better procedure for image compression. The various parameters used in this work are :

Signal to noise ratio

Peak signal to noise ratio

Compression ratio

Relative redundancy

Bits per pixel of the compressed image

Table 2: Comparison between conventional techniques and artificial neural networks approach.

\begin{tabular}{|l|c|c|c|c|c|}
\hline $\begin{array}{l}\text { ALGO- } \\
\text { RITHM }\end{array}$ & SNR & PSNR & CR & RD & B/P \\
\hline RLE & 0.021 & 5.373 & 5.395 & 0.815 & 0.704 \\
\hline BTC & 12.792 & 18.144 & 3.475 & 0.712 & 1.089 \\
\hline DCT & 20.870 & 26.222 & 3.514 & 0.716 & 1.077 \\
\hline $\begin{array}{l}\text { LM } \\
\text { (ANN) }\end{array}$ & 16.945 & 22.296 & 5.880 & 0.830 & 0.824 \\
\hline
\end{tabular}

\subsection{Observations}

- In the run length encoding algorithm the signal to noise ratio was significantly low (SNR).

- The best signal to noise (SNR) ratio was achieved by using discrete cosine transformation.

- $\quad$ Run length encoding detected a comparatively low peak signal to noise ratio (PSNR).

- Discrete cosine transformation achieved the maximum peak signal to noise ratio (PSNR).

- The maximum compression ratio (CR) was given by Levenberg-Marquardt algorithm.

- Block truncation coding achieved the lowest compression ratio (CR).

- The lowest relative redundancy was achieved by block truncation coding. 
- Levenberg-Marquardt algorithm had the maximum relative redundancy.

- Lowest bits per pixel were given by run length encoding.

- Block truncation coding provide the maximum bits per pixel.

\section{CONCLUSION}

Image compression can be done using the conventional techniques available. Here, in this work an attempt is being made to perform image compression using the artificial neural networks approach and an experimental comparison is done between conventional techniques and artificial neural network approach for image compression. We can conclude from the observed values of our experimental study, that the artificial neural network approach when applied to image compression can provide better results than compared to the conventional techniques. There are various applications of image compression such as, to reduce the storage space of an image or to easily transfer images over a network. The comparative results in this work indicates that artificial neural network approach has the potential for performing image compression. There are various learning algorithms. In this work, image compression has been performed with only one learning algorithm, i.e. Levenberg-Marquardt algorithm, and is compared with conventional methods. As a future scope, image compression can be performed with various learning algorithms for more number of images.

\section{REFERENCES}

[1] Manjinder Kaur, Gaganpreet Kaur, "A Survey of Lossless and Lossy Image Compression Techniques", International Journal of Advanced Research in Computer Science and Software Engineering, 2013.

[2] Doaa Mohammed, Fatma Abou-Chadi, "Image Compression Using Block Truncation Coding", International Journal of Electronics and Computer Science Engineering, 2011.

[3] Bhawna Gautam. May, 2010. Image Compression Using Discrete Cosine Transform \& Discrete Wavelet Transform. National Institute of Technology, Rourkela.

[4] Anjana B ,Mrs Shreeeja R "Image compression: an artificial neural network approach". Vol.2, issue 8, 2012.

[5] Pranob K Charles, Dr. H.Khan, Ch.Rajesh Kumar, N.Nikhita Santhosh Roy, V.Harish,M.Swathi , "Artificial Neural Network based Image Compression using Levenberg- Marquardt Algorithm”, International Journal of Modern Engineering Research (IJMER) , 2013

[6] Venkata Rama Prasad Vaddella, "Artificial neural networks for compression of digital images: a review", International Journal of Reviews in Computing, 20092010 . 